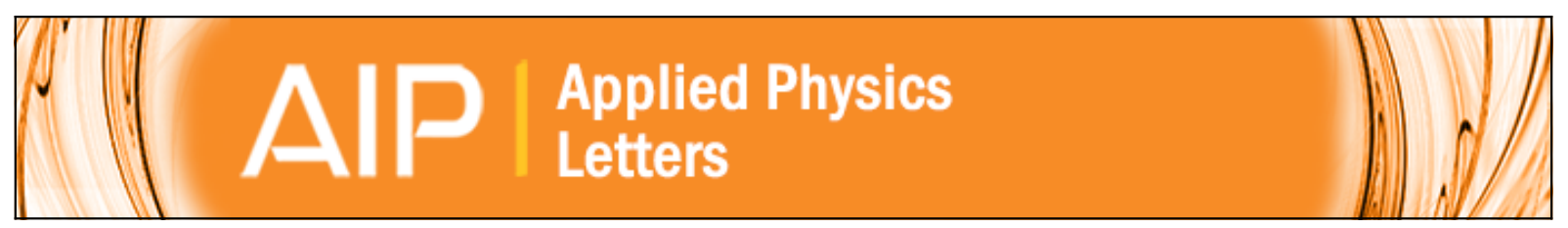

\title{
Single photon emission up to liquid nitrogen temperature from charged excitons confined in GaAs-based epitaxial nanostructures
}

Ł. Dusanowski, M. Syperek, A. Maryński, L. H. Li, J. Misiewicz, S. Höfling, M. Kamp, A. Fiore, and G. Sęk

Citation: Applied Physics Letters 106, 233107 (2015); doi: 10.1063/1.4922455

View online: http://dx.doi.org/10.1063/1.4922455

View Table of Contents: http://scitation.aip.org/content/aip/journal/apl/106/23?ver=pdfcov

Published by the AIP Publishing

\section{Articles you may be interested in}

Volmer-Weber InAs quantum dot formation on InP (113)B substrates under the surfactant effect of Sb

Appl. Phys. Lett. 105, 033113 (2014); 10.1063/1.4891505

Nanotemplate-directed InGaAs/GaAs single quantum dots: Toward addressable single photon emitter arrays J. Vac. Sci. Technol. B 32, 02C106 (2014); 10.1116/1.4863680

Long wavelength $(>1.55 \mu \mathrm{m})$ room temperature emission and anomalous structural properties of InAs/GaAs quantum dots obtained by conversion of In nanocrystals

Appl. Phys. Lett. 102, 073103 (2013); 10.1063/1.4792700

Single quantum dot emission at telecom wavelengths from metamorphic InAs/lnGaAs nanostructures grown on GaAs substrates

Appl. Phys. Lett. 98, 173112 (2011); 10.1063/1.3584132

InGaAs/GaAs quantum nanostructure fabrication on GaAs (111)A vicinal substrates by atomic layer epitaxy J. Appl. Phys. 83, 5525 (1998); 10.1063/1.367406

LIVE DEMO

The Basics of COMSOL in 18 Minutes

$\checkmark$ COMSOL

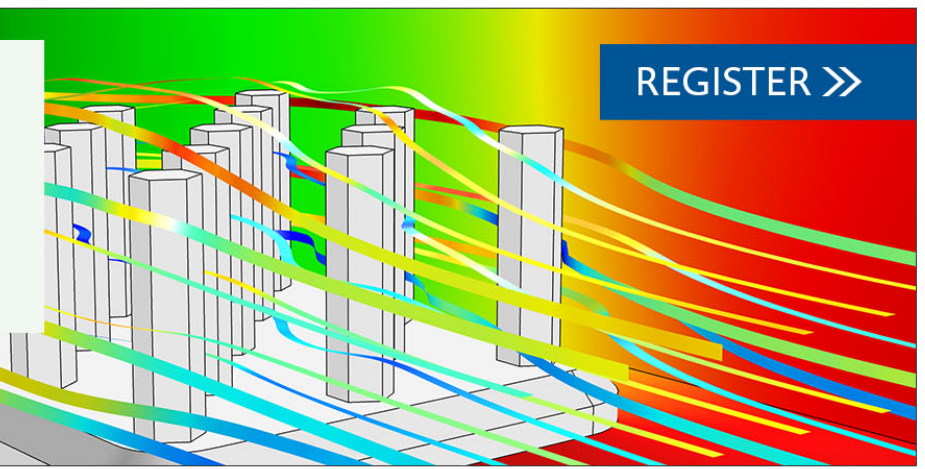




\title{
Single photon emission up to liquid nitrogen temperature from charged excitons confined in GaAs-based epitaxial nanostructures
}

\author{
Ł. Dusanowski, ${ }^{1, a)}$ M. Syperek, ${ }^{1}$ A. Maryński, ${ }^{1}$ L. H. Li, ${ }^{2, b)}$ J. Misiewicz, ${ }^{1}$ S. Höfling, ${ }^{3,4}$ \\ M. Kamp, ${ }^{3}$ A. Fiore, ${ }^{2,5}$ and G. Sęk ${ }^{1}$ \\ ${ }^{1}$ Laboratory for Optical Spectroscopy of Nanostructures, Department of Experimental Physics, \\ Wrocław University of Technology, Wybrzeże Wyspiańskiego 27, 50-370 Wrocław, Poland \\ ${ }^{2}$ Ecole Polytechnique Fédérale de Lausanne, Institute of Quantum Electronics and Photonics, Station 3 , \\ CH-1015 Lausanne, Switzerland \\ ${ }^{3}$ Technische Physik \& Wilhelm-Conrad-Röntgen-Research Center for Complex Material Systems, \\ University of Würzburg, Am Hubland, D-97074 Würzburg, Germany \\ ${ }^{4}$ SUPA, School of Physics and Astronomy, University of St. Andrews, North Haugh, KY16 9SS St. Andrews, \\ United Kingdom \\ ${ }^{5}$ COBRA Research Institute, Eindhoven University of Technology, PO Box 513, 5600 MB Eindhoven, \\ The Netherlands
}

(Received 13 January 2015; accepted 1 June 2015; published online 11 June 2015)

\begin{abstract}
We demonstrate a non-classical photon emitter at near infrared wavelength based on a single (In,Ga)As/GaAs epitaxially grown columnar quantum dot. Charged exciton complexes have been identified in magneto-photoluminescence. Photon auto-correlation histograms from the recombination of a trion confined in a columnar dot exhibit sub-Poissonian statistics with an antibunching dip yielding $\mathrm{g}^{(2)}(0)$ values of 0.28 and 0.46 at temperature of 10 and $80 \mathrm{~K}$, respectively. Our experimental findings allow considering the GaAs-based columnar quantum dot structure as an efficient single photon source operating at above liquid nitrogen temperatures, which in some characteristics can outperform the existing solutions of any material system. (C) 2015 AIP Publishing LLC.
\end{abstract}

[http://dx.doi.org/10.1063/1.4922455]

The rapidly evolving field of secure quantum communication, data transmission, and processing on the single photon level requires utilization of the single photon source (SPS). ${ }^{1-3}$ It has been proven that a semiconductor quantum dot (QD) can constitute a key element of an SPS since it provides generation of single photons with high internal quantum efficiency and long-term stability, narrow spectral linewidths, and high generation rates in comparison to other solutions such as cold atom or ion in a trap, single molecules, color centers in diamond, or nanocrystals. ${ }^{4}$ In case of applications where coherence between single photons is required, the emitter has to be very well protected from fluctuations in its environment. This condition is fulfilled only by two classes of emitters, i.e., isolated atoms or ions in traps in ultra-vacuum and molecules or self-assembled quantum dots in solid matrices at cryogenic temperatures. ${ }^{4}$ In case of the latter, III-V semiconductor nanostructures are especially advantageous due to the well-established micro-fabrication technology and ability to be integrated with today's complex photonic systems. ${ }^{1-3,5}$ However, sensitivity of the emission efficiency to temperature prevents their practical implementation. Generation of single photons at elevated temperatures has been achieved by utilizing QDs made of wide-bandgap II-VI materials (up to $\mathrm{T}=220 \mathrm{~K}$ with $(\mathrm{Cd}, \mathrm{Zn}) \mathrm{Se} / \mathrm{ZnSe} \mathrm{QD},{ }^{6}$ and $300 \mathrm{~K}$ for $\left.\mathrm{CdSe} /(\mathrm{Zn}, \mathrm{S}) \mathrm{Se} / \mathrm{MgS} \mathrm{QD}^{7}\right)$, group-III nitrides (up to $300 \mathrm{~K}$ for $\mathrm{GaN} / \mathrm{AlN} \mathrm{QD}^{8}$ ), and some of other III-V material systems (InP/(In,Al)GaP at $80 \mathrm{~K}$ (Ref. 9) and GaAs/

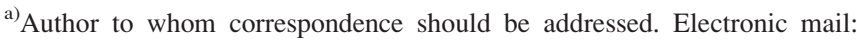
lukasz.dusanowski@pwr.edu.pl.

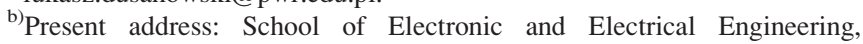
University of Leeds, Leeds LS2 9JT, United Kingdom.
}

(Al,Ga)As QD at $80 \mathrm{~K}$ (Ref. 10)). However, these solutions offer predominantly operation in the visible spectral range. In contrast, a proof of a single photon emission at liquid nitrogen temperature for the $\mathrm{In}(\mathrm{Ga}) \mathrm{As} / \mathrm{GaAs}$ QD system, which could allow to construct devices for longer wavelengths, has been shown in a very few cases only ${ }^{11,12}$ and based on a neutral exciton emission. Nevertheless, the (In,Ga)As/GaAs QD is still considered as a competitive solution. By exploiting such material system, the SPS emission wavelength can be pushed towards the near-infrared spectral region, up to even $1.3-1.55 \mu \mathrm{m} .{ }^{13,14}$ In addition, the mature GaAs-based technology permits producing more sophisticated and multifunctional quantum systems, including photonic waveguides, microcavities with exceptional parameters, or photonic integrated circuits. ${ }^{15-18}$ All these can compensate for the disadvantage of the temperature impact.

In this letter, we investigate the potential of a characteristic nanostructure, an (In,Ga)As/GaAs columnar QD (CQD), as single-photon emitter at elevated temperatures. We report single-photon emission at $\mathrm{T}=80 \mathrm{~K}$ from a charged exciton confined in an optically pumped CQD, where the character of a charged complex has been confirmed in magnetophotoluminescence (PL) experiments. The charged exciton is potentially more interesting as its maximum achievable emission rate is not limited by dark states, in contrast to an exciton. ${ }^{19}$ Yet, single photon emission from charged exciton complexes (free of dark states) in GaAs system has only been reported below liquid nitrogen temperatures. ${ }^{19-22}$

By performing systematic studies of PL and measuring the photon emission statistics as a function of temperature, we demonstrate that such kind of nanostructures can be 
considered as efficient near-infrared single photon emitters able to operate at liquid nitrogen temperature. The unique structure of CQDs makes it possible to tailor the built-in strain field and the quantum confinement. ${ }^{23,24}$ This in turn can allow manipulating the emitter properties via intentionally forming the light-hole exciton, for instance, enabling the control of the polarization properties, ${ }^{25,26}$ engineer the exciton dipole moment ${ }^{25}$ crucial for optical Stark effect tuning, ${ }^{27}$ or it could lead to the reduction of the exciton fine structure splitting. ${ }^{28}$ Consequently, it may be possible to consider a CQD structure not only as a well-controlled single photon emitter with broad range of tunable parameters but also as a potential source of polarization-entangled photon pairs.

The CQDs were fabricated by molecular beam epitaxy through a periodic overgrowth of InAs and GaAs ultrathin layers (a kind of superlattice-SL) on a (001)-oriented GaAs substrate. ${ }^{29}$ This kind of growth technique permits for a very precise control of the nanostructure's height, indium composition profile along the growth direction, and built-in strain field by varying the SL period. ${ }^{29,30}$ In consequence, it allowed forming epitaxially rod-like or post-like quantum nanostructures varying in height to base width aspect ratio from 0.5 up to 4 , and beyond. ${ }^{29,30}$ The structure under study is made by initially depositing the seeding layer of InAs QDs on a GaAs buffer in a self-assembled manner upon the Stranski-Krastanow growth conditions. Next, a short period $\mathrm{SL}$ is deposited in three times repeated sequence of $0.62 \mathrm{ML}$ of InAs and $3 \mathrm{ML}$ of GaAs. The in-plane strain distribution created by the first QD layer favors the In incorporation on top of QDs, resulting in In-rich column-shaped nanostructures with height to base width ratio in the range of 0.5-1.0. Based on the transmission electron microscope images, the In content along the CQD is around $40 \%$ and in the surrounding two-dimensional "immersion" layer is $\sim 15 \%{ }^{29,30}$ The structure is nominally undoped. More details of the growth process have already been reported elsewhere. ${ }^{30}$ For single dot spectroscopy, a set of mesas with sizes varying from 0.1 to $2 \mu \mathrm{m}$ have been created on the sample surface by combining electron beam lithography and reactive ion etching.

For all experiments, the CQD structure was kept in a liquid-helium continuous-flow optical cryostat equipped with a superconducting magnetic coil. The system capabilities allow changing sample temperature in the range of $5-300 \mathrm{~K}$, and magnetic field up to $\mathrm{B}=5 \mathrm{~T}$. Single emission lines from CQD structures have been measured upon nonresonant excitation with a $787 \mathrm{~nm}$ line of a continuous wave (CW) semiconductor laser. For this purpose, spatially resolved PL $(\mu \mathrm{PL})$ signal was collected by a microscope objective with numerical aperture $(\mathrm{NA})=0.4$ and then spectrally dispersed in a $0.3 \mathrm{~m}$-focal-length monochromator combined with a nitrogen-cooled InGaAs linear array detector. The radius of the diffraction limited excitation spot and the spectral resolution are $\sim 2 \mu \mathrm{m}$ and $\sim 100 \mu \mathrm{eV}$, respectively. The photon auto- and cross-correlation experiments were conducted with a Hanbury Brown and Twiss interferometer. ${ }^{31}$ In this case, a second $0.3 \mathrm{~m}$-focal-length monochromator was used as an additional spectral filter for selected emission lines. Each of the monochromators' was equipped with the Si-based avalanche photodiode (APD) with $\sim 15 \%$ quantum efficiency and $\sim 25$ dark counts per second in the considered spectral range. A multichannel picosecond event timer acquired the photon correlation statistics. The influence of the magnetic field on single emission lines for CQD structures has been examined in a slightly modified configuration of the described $\mu \mathrm{PL}$ setup, equipped with a $1 \mathrm{~m}$-focal-length monochromator providing spectral resolution on the level of $25 \mu \mathrm{eV}$. The magnetic field vector was oriented perpendicular to the structure growth axis (z-axis): $\mathrm{B} \perp z$ (the Voigt configuration). The polarization state of emitted photons was analyzed by using quarter wave-plate and linear polarizer placed in the front of the monochromator entrance. All experiments have been performed on several emission lines indicating high emission intensities at elevated temperatures. Since measurements were performed on the sample with a well-defined mesa structure, it was possible to run experiments repeatedly on the same CQD within a chosen mesa even within several days or weeks interval. Multiple microphotoluminescence and photon correlation experiments were performed for the same dots and emission lines with a good reproducibility.

Figure 1 shows $\mu \mathrm{PL}$ spectra consisting of a few wellisolated emission lines from several CQDs enclosed in the mesa structure of $200 \times 200 \mathrm{~nm}^{2}$ size. Low temperature $(\mathrm{T}=5 \mathrm{~K})$ emission presented in Fig. 1(a) is recorded under the excitation pumping power of about $20 \mu \mathrm{W}$ (measured outside the cryostat). It presents only few spectral features appearing in the energy range of $1.270-1.282 \mathrm{eV}$. Nevertheless, the overall $\mu \mathrm{PL}$ spectrum is dominated by a

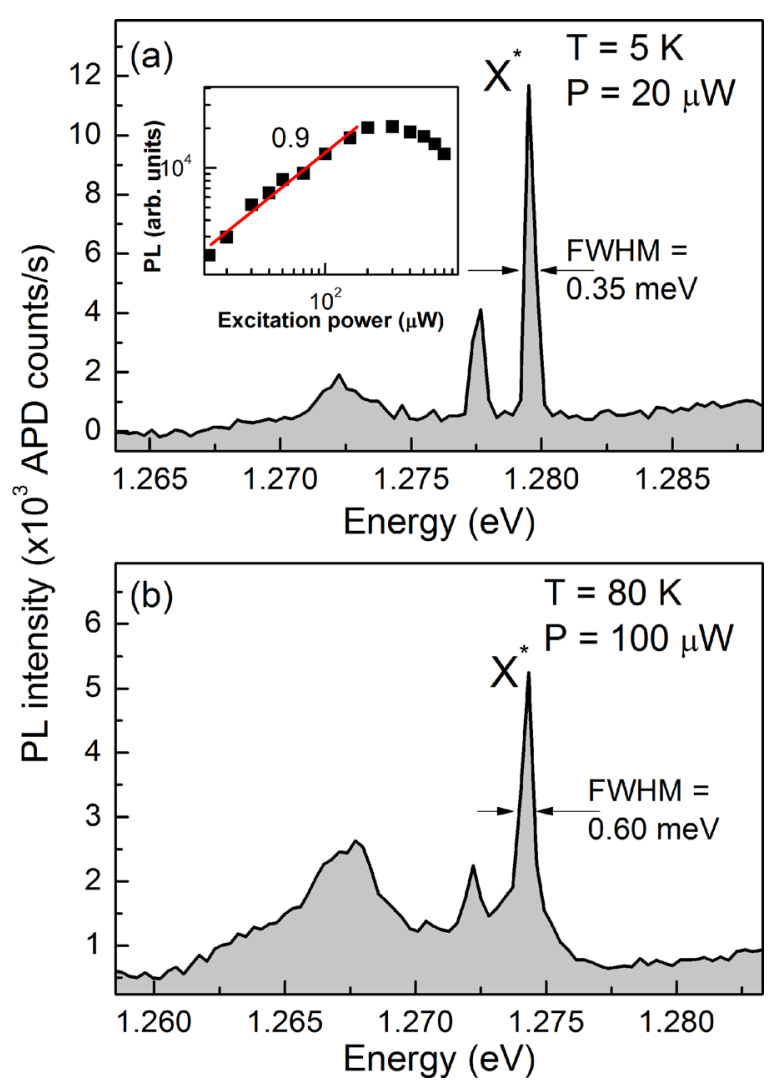

FIG. 1. Emission spectra from single mesa structure with InGaAs/GaAs CQDs measured at (a) $5 \mathrm{~K}$, and (b) $80 \mathrm{~K}$ with excitation powers equal to 20 $\mu \mathrm{W}$ and $100 \mu \mathrm{W}$, respectively. Inset: PL intensity dependence on excitation power in $\log$ scale for a selected emission line assigned as $\mathrm{X}^{*}$. 
single and intense emission line (labeled as $\mathrm{X}^{*}$ ) with a full width at half maximum of $0.35 \mathrm{meV}$ centered at $1.279 \mathrm{eV}$. The spectrum in Fig. 1(a) on the left hand side of the $\mathrm{X}^{*}$ line consists of several less intensive emission lines. In order to check if those lines originate from the same dot as the $\mathrm{X}^{*}$ line, we measured the respective photon cross-correlations, but no clear dependencies were obtained, suggesting emission from individual CQDs enclosed within the same mesa. The intensity of the $\mathrm{X}^{*}$ line as a function of the excitation power exhibits a nearly linear dependence at low excitation-see inset in Fig. 1. This is characteristic for neutral or charged exciton emission, but not for a biexciton where it should be rather super-linear. No energy splitting of the $X^{*}$ line in the cross-linearly polarized photoluminescence signal (Fig. 2(a)) within the spectral resolution of about $25 \mu \mathrm{eV}$ may indicate a lack of the fine structure splitting and thus suggests the charged exciton (a trion) character of this line.

In order to unambiguously clarify the origin of the $\mathrm{X}^{*}$ spectral feature, we performed $\mu \mathrm{PL}$ studies under the magnetic field applied in the Voigt configuration ( $\mathrm{B} \perp z$, where $z$ is the growth direction). Figure 2(b) presents $\mu \mathrm{PL}$ spectra of the $\mathrm{X}^{*}$ line at $\mathrm{B}=5.0 \mathrm{~T}$. The spectra are recorded under two cross-linear polarizations of the detected photons: "horizontal"- $\mathrm{H}$ (red circles in Fig. 2), and "vertical"- $-\mathrm{V}$
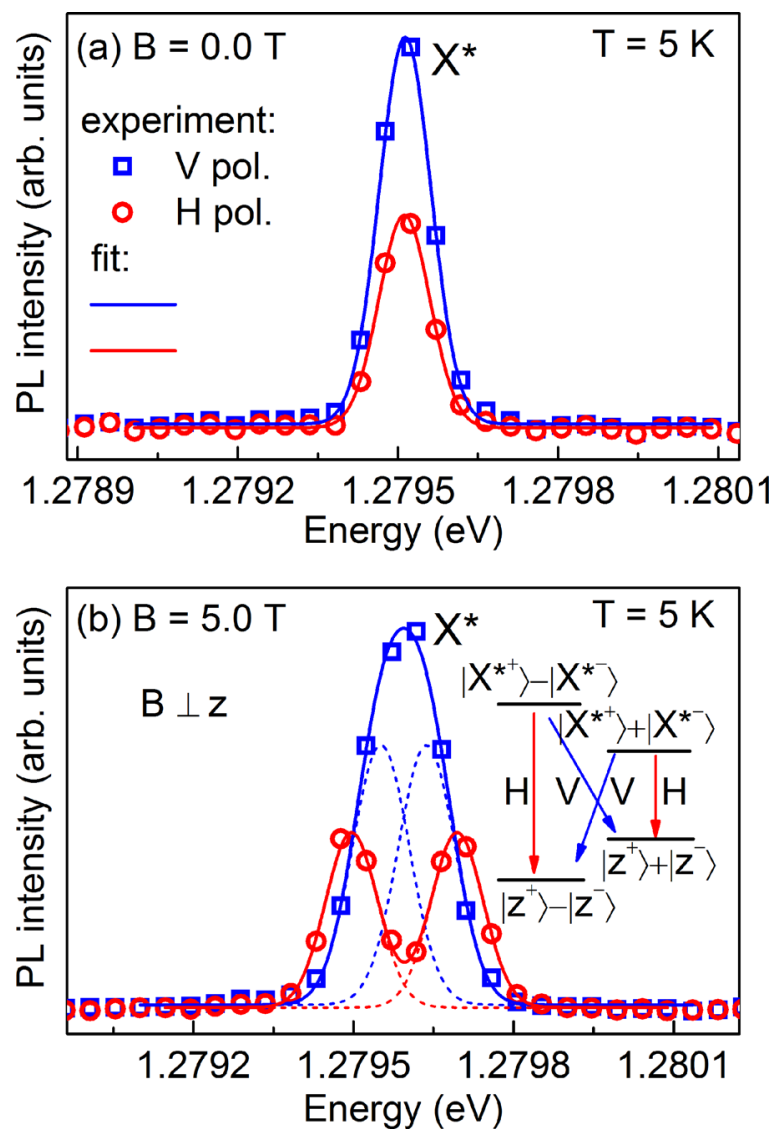

FIG. 2. Emission spectra from single charged exciton line $\left(\mathrm{X}^{*}\right)$ recorded at two linear polarizations: horizontal $(\mathrm{H})$ and vertical $(\mathrm{V})$ in case of (a) no magnetic field applied and (b) magnetic field $\mathrm{B}=5.0 \mathrm{~T}$ applied perpendicularly to growth axis $z$ (Voigt configuration). Inset: Diagram of allowed charged exciton transitions in the basis perpendicular to $z$ axis in case of $\mathrm{B} \perp z$, where $\left|X^{*+}\right\rangle \pm\left|X^{*-}\right\rangle$ and $\left|z^{+}\right\rangle \pm\left|z^{-}\right\rangle$are mixed trion and single particle (electron or heavy hole) spin states, consequently, polarized along the growth axis. (blue squares in Fig. 2). The "center-of-mass" of the emission peak position is shifted by $\sim 80 \mu \mathrm{eV}$ with respect to $\mathrm{B}=0.0 \mathrm{~T}$ due to the diamagnetic effect. In the $\mathrm{H}$ polarization, a doublet of lines is clearly observed with the energy splitting of $\sim 200 \mu \mathrm{eV}$. In the $\mathrm{V}$ polarization, the $\mathrm{X}^{*}$ line is significantly broadened when compared to the one observed at the zero magnetic field, which may indicate its fine structure. Indeed, the $\mathrm{X}^{*}$ line can be very well decomposed into two Gaussian profiles (see dashed lines under the V polarized emission in Fig. 2(b)). The fitting procedure gave the splitting on the level of $100 \mu \mathrm{eV}$. The registered set of optical transitions corresponds perfectly to the spin-dependent optical selection rules for the charged exciton state under the magnetic field in the Voigt geometry (see the sketch in Fig. 2(b)). Therefore, the observation of the cross-linearly polarized pairs of doublets evidences that the $\mathrm{X}^{*}$ emission line originates from the charged exciton recombination process. ${ }^{21,32}$ It is worth mentioning that in case of emission from a neutral exciton at non-zero $\mathrm{B}(\mathrm{B} \perp z)$, one would expect to observe split pairs of opposite-circularly polarized lines and possibly additional pair of lines from the so-called dark exciton states, ${ }^{21,32}$ which is not the case here.

Increasing the temperature of the CQD structure up to $80 \mathrm{~K}$ transforms the $\mu \mathrm{PL}$ spectrum from Fig. 1(a) to that shown in Fig. 1(b). In this case, the excitation pumping power was slightly elevated up to $100 \mu \mathrm{W}$ in order to keep reasonable emission intensity, i.e., to partially compensate the temperature-driven carrier losses in CQDs. The $\mathrm{X}^{*}$ emission feature still dominates the $\mu \mathrm{PL}$ spectrum - the peak is shifted to the red by $\sim 5.2 \mathrm{meV}$ and the line is broadened up to $0.6 \mathrm{meV}$. While the energy shift is mainly driven by the change in the energy gap of the CQD material, the broadening of the emission line is related to the increase of the phonon reservoir and therefore enhanced the exciton-acoustic phonon coupling in the system. ${ }^{33}$

The full picture of the temperature evolution of the $\mathrm{X}^{*}$ emission line in the range of $5-80 \mathrm{~K}$ is presented in Fig. 3. These spectra have been recorded each at the same experimental conditions, in particularly the same $\mathrm{CW}$ laser excitation power of $40 \mu \mathrm{W}$. For clarity, each spectrum is normalized to maximum intensity. With increasing the temperature, the main emission peak shows clearly a redshift and the thermally activated intensity quenching (inset in Fig. $3)$.

Once the $\mathrm{X}^{*}$ line can be tracked in the $\mu \mathrm{PL}$ spectrum at elevated temperatures, it is possible to measure the autocorrelation statistics of the emission process in the entire temperature range in order to evaluate the non-classicality of the emitter. Figure 4 shows examples of auto-correlation histogram recorded at $\mathrm{T}=10 \mathrm{~K}$ (Fig. 4(a)), $50 \mathrm{~K}$ (Fig. 4(b)), and $80 \mathrm{~K}$ (Fig. 4(c)). In the case of photon correlation measurements in order to improve the counts number reduced due to thermal quenching of the radiative efficiency, the excitation power has been increased accordingly. In general, the impact of raising the lattice temperature and excitation power is reflected in the shape of the collected histograms that resemble the $\mathrm{g}^{(2)}(\tau)$ function. The built-up rate of the $\mathrm{g}^{(2)}(\tau)$ function in the case of the $\mathrm{CW}$ excitation represents occupation changes of a certain state of an emitter considered as a twolevel system. The changes are driven by the carrier 


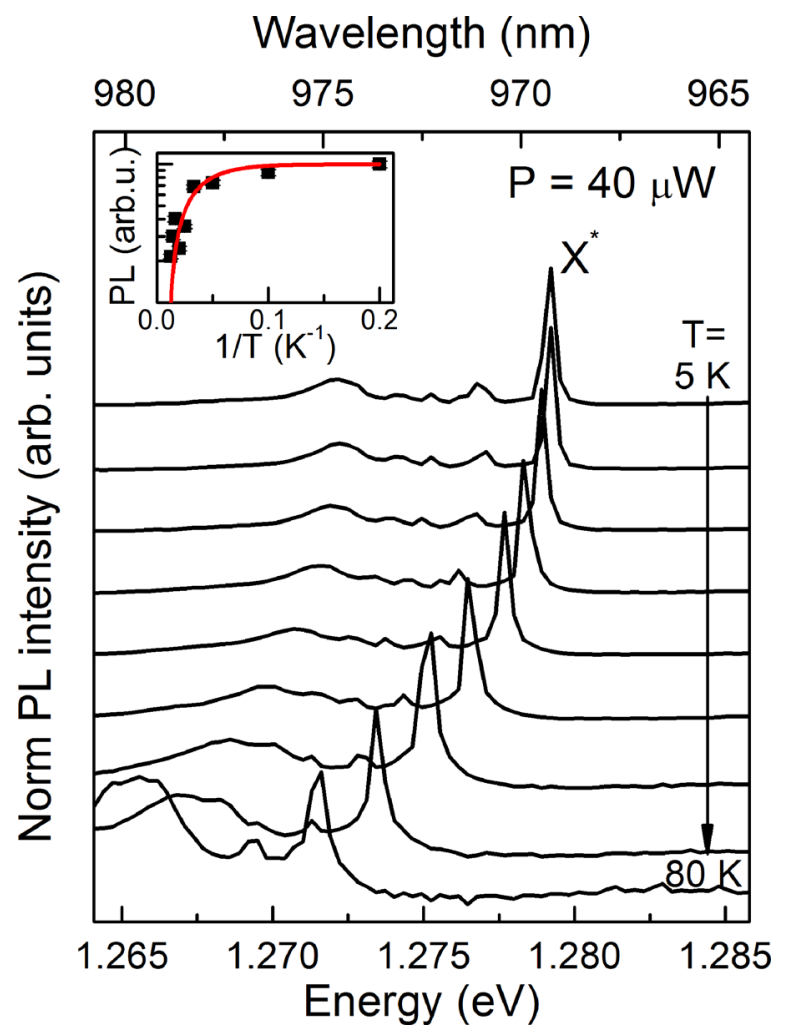

FIG. 3. Normalized to maximum intensity temperature dependence of the PL spectra from single mesa structure with InGaAs/GaAs CQDs in the range of $5-80 \mathrm{~K}$ recorded at excitation power equal to $40 \mu \mathrm{W}$. Inset: Evolution of the $\mathrm{X}^{*}$ line integrated intensity with temperature.

generation via photoexcitation, radiative recombination of a certain state, and non-radiative carrier losses induced by the temperature. Those effects contribute to observed narrowing of the $\mathrm{g}^{(2)}(\tau)$ dip; however, they should not strongly affect the $g^{(2)}(0)$ value in the considered range of parameters. One can see in Fig. 4 that all the three graphs present a clear antibunching dip in the auto-correlation function at zero delay times with the as-measured $\mathrm{g}^{(2)}(0)$ value equal to 0.32 $(\mathrm{T}=10 \mathrm{~K}), 0.35(\mathrm{~T}=50 \mathrm{~K})$, and $0.46(\mathrm{~T}=80 \mathrm{~K})$. After including the PL background counts correction, ${ }^{34}$ these values can further be reduced down to $0.26,0.28$, and 0.29 , respectively. The non-zero value of $g^{(2)}(0)$ is most likely due to the combination of setup limited time-resolution $(\sim 700$ ps) and large time bin between the points (256 ps). Taking into account the abovementioned via performing the deconvolution procedure as in Ref. 35 , the $g^{(2)}(0)$ turns out to be close to zero for all the cases (blue dashed line on Fig. 4). The used continues-flow liquid helium cryostat allowed the observation of single photons generated from CQDs at temperatures up to $80 \mathrm{~K}$ even after many heat-up and cool-down cycles. In this sense, the optically driven CQD single photon emitter remains a system isolated from the external conditions including temperature. Therefore, it could also allow integration of such nanostructures with a more compact lowvibrational cryocooler ${ }^{36}$ or even a simple liquid nitrogen cooled cryostat in order to create a robust and environment insensitive, efficient, and fully operational single photon source device.

In conclusion, we have investigated the temperature dependence of emission processes from a single (In,Ga)As/

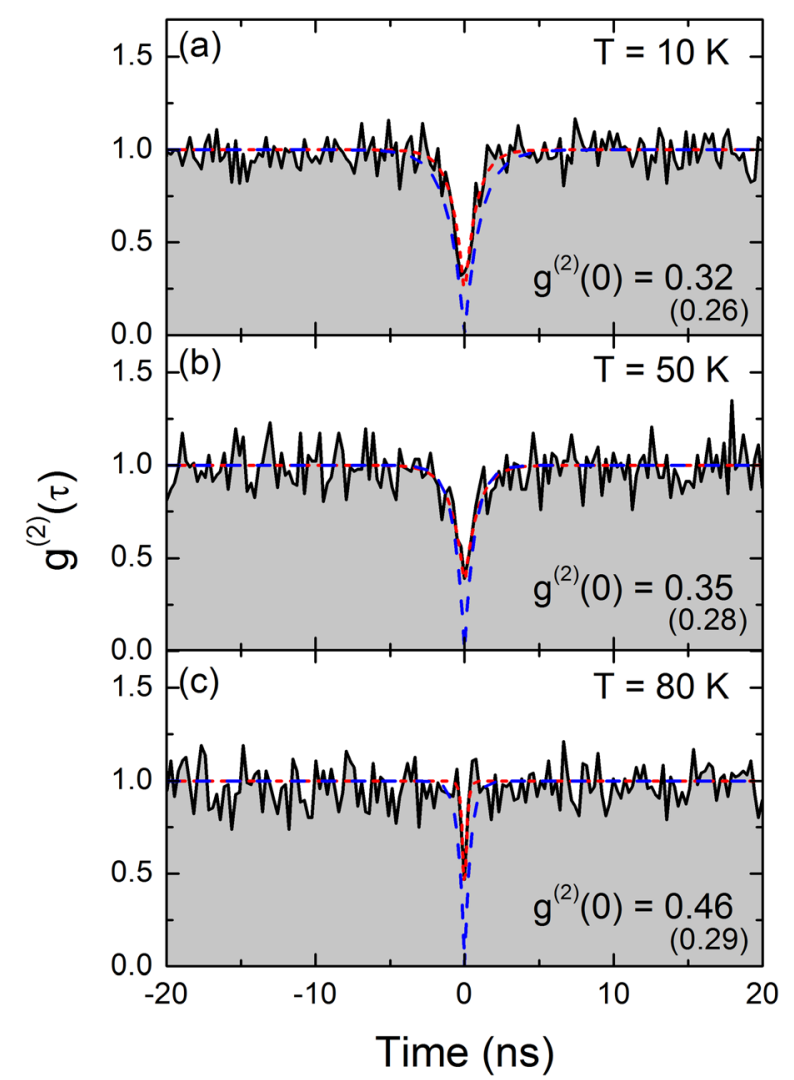

FIG. 4. Auto-correlation coincidence counts histogram measured at (a) $10 \mathrm{~K}$, (b) $50 \mathrm{~K}$, and (c) $80 \mathrm{~K}$ from the same charged exciton line $\mathrm{X}^{*}$. Dashed red line is exponential $g^{(2)}$ fit to experimental data; dashed blue line is $g^{(2)}(\tau)$ function found upon the deconvolution procedure. $g^{(2)}(0)$ values in parenthesis are corrected for background dark counts.

GaAs columnar quantum dot by means of high spatial resolution photoluminescence, magneto-photoluminescence, and photon correlation spectroscopy. We have demonstrated a single photon emitter based on a charged exciton state confined in a columnar quantum dot. The emitter, driven non-resonantly by the $\mathrm{CW}$ laser source, preserves its quantum nature of the emission process up to at least $\mathrm{T}=80 \mathrm{~K}$. The $g^{(2)}(0)$ value significantly below the 0.5 limit indicates that the charge exciton state confined in the CQD can be considered as a true single photon generator able to operate above the liquid nitrogen temperature. This observation is a promising result in the context of further research on triggered single photon sources made within the GaAstechnology, especially when regarding the integration with silicon, ${ }^{37}$ silicon-on-insulator, ${ }^{38}$ or germanium-on-insulator $^{39}$ substrates in order to create hybrid platforms for the development of future high-performance CMOS-based photonic systems ${ }^{40}$ or photonic MEMS. ${ }^{41}$ Utilization of considered in this letter CQD nanostructures with compact cooling system could allow to create a robust, handy, and fully operational single photon source device for quantum cryptography protocols like, for example, free-space quantum key distribution. ${ }^{4,42}$ Moreover, thanks to the well-established III-V semiconductor technology, CQD single photon emitters could be also applied in more complex and multifunctional quantum systems such as photonic waveguides, microcavities, or photonic integrated circuits. ${ }^{15-18}$ 
This research was supported by the National Science Center Grant No. 2011/01/B/ST3/02379. The experiments have partly been performed within the NLTK infrastructure, Project No. POIG. 02.02.00-003/08-00. We acknowledge M. Pieczarka for his technical assistance in performing some of the spectroscopic measurements. S.H. gratefully acknowledges support by the Royal Society and the Wolfson Foundation.

${ }^{1}$ Single Quantum Dots: Fundamentals, Applications, and New Concepts, edited by P. Michler (Springer-Verlag, Berlin, Heidelberg, 2003).

${ }^{2}$ S. Buckley, K. Rivoire, and J. Vučković, Rep. Prog. Phys. 75, 126503 (2012).

${ }^{3}$ G.-C. Shan, Z.-Q. Yin, C. H. Shek, and W. Huang, Front. Phys. 9, 170 (2013).

${ }^{4}$ B. Lounis and M. Orrit, Rep. Prog. Phys. 68, 1129 (2005).

${ }^{5}$ K. Asakawa, Y. Sugimoto, Y. Watanabe, N. Ozaki, A. Mizutani, Y. Takata, Y. Kitagawa, H. Ishikawa, N. Ikeda, K. Awazu, X. Wang, A. Watanabe, S. Nakamura, S. Ohkouchi, K. Inoue, M. Kristensen, O. Sigmund, P. I. Borel, and R. Baets, New J. Phys. 8, 208 (2006).

${ }^{6}$ A. Tribu, G. Sallen, T. Aichele, R. André, J. P. Poizat, C. Bougerol, S. Tatarenko, and K. Kheng, Nano Lett. 8, 4326 (2008).

${ }^{7}$ O. Fedorych, C. Kruse, A. Ruban, D. Hommel, G. Bacher, and T. Kümmell, Appl. Phys. Lett. 100, 061114 (2012).

${ }^{8}$ M. J. Holmes, K. Choi, S. Kako, M. Arita, and Y. Arakawa, Nano Lett. 14, 982 (2014).

${ }^{9}$ M. Reischle, G. J. Beirne, W. M. Schulz, M. Eichfelder, R. Rosbach, M. Jetter, and P. Michler, Opt. Express 16, 12771 (2008).

${ }^{10}$ L. Cavigli, S. Bietti, N. Accanto, S. Minari, M. Abbarchi, G. Isella, C. Frigeri, A. Vinattieri, M. Gurioli, and S. Sanguinetti, Appl. Phys. Lett. 100, 231112 (2012)

${ }^{11}$ R. P. Mirin, Appl. Phys. Lett. 84, 1260 (2004).

${ }^{12}$ X. M. Dou, X. Y. Chang, B. Q. Sun, Y. H. Xiong, Z. C. Niu, S. S. Huang, H. Q. Ni, Y. Du, and J. B. Xia, Appl. Phys. Lett. 93, 101107 (2008).

${ }^{13}$ B. Alloing, C. Zinoni, V. Zwiller, L. H. Li, C. Monat, M. Gobet, G. Buchs, A. Fiore, E. Pelucchi, and E. Kapon, Appl. Phys. Lett. 86, 101908 (2005).

${ }^{14}$ E. S. Semenova, R. Hostein, G. Patriarche, O. Mauguin, L. Largeau, I. Robert-Philip, A. Beveratos, and A. Lemaître, J. Appl. Phys. 103, 103533 (2008).

${ }^{15}$ J. P. Reithmaier, G. Sęk, A. Loffler, C. Hofmann, S. Kuhn, S. Reitzenstein, L. Keldysh, V. Kulakovskii, T. Reinecke, and A. Forchel, Nature 432, 197 (2004).

${ }^{16}$ A. Dousse, J. Suffczyński, A. Beveratos, O. Krebs, A. Lemaître, I. Sagnes, J. Bloch, P. Voisin, and P. Senellart, Nature 466, 217 (2010).

${ }^{17}$ S. Fattah poor, T. B. Hoang, L. Midolo, C. P. Dietrich, L. H. Li, E. H. Linfield, J. F. P. Schouwenberg, T. Xia, F. M. Pagliano, F. W. M. van Otten, and A. Fiore, Appl. Phys. Lett. 102, 131105 (2013).

${ }^{18}$ T. Ba Hoang, J. Beetz, L. Midolo, M. Skacel, M. Lermer, M. Kamp, S. Höfling, L. Balet, N. Chauvin, and A. Fiore, Appl. Phys. Lett. 100, 061122 (2012).

${ }^{19}$ S. Strauf, N. G. Stoltz, M. T. Rakher, L. A. Coldren, P. M. Petroff, and D. Bouwmeester, Nat. Photonics 1, 704 (2007).
${ }^{20}$ S. M. Ulrich, M. Benyoucef, P. Michler, N. Baer, P. Gartner, F. Jahnke, M. Schwab, H. Kurtze, M. Bayer, S. Fafard, Z. Wasilewski, and A. Forchel, Phys. Rev. B 71, 235328 (2005).

${ }^{21}$ M. Bayer, G. Ortner, O. Stern, A. Kuther, A. A. Gorbunov, A. Forchel, P. Hawrylak, S. Fafard, K. Hinzer, T. L. Reinecke, S. N. Walck, J. P. Reithmaier, F. Klopf, and F. Schäfer, Phys. Rev. B 65, 195315 (2002).

${ }^{22}$ T. Heindel, C. Schneider, M. Lermer, S. H. Kwon, T. Braun, S. Reitzenstein, S. Höfling, M. Kamp, and A. Forchel, Appl. Phys. Lett. 96, 011107 (2010).

${ }^{23}$ J. Andrzejewski, G. Sęk, E. O’Reilly, A. Fiore, and J. Misiewicz, J. Appl. Phys. 107, 073509 (2010).

${ }^{24}$ P. Ridha, L. H. Li, M. Mexis, P. M. Smowton, J. Andrzejewski, G. Sek, J. Misiewicz, E. P. O'Reilly, G. Patriarche, and A. Fiore, IEEE J. Quantum Electron. 46, 197 (2010).

${ }^{25}$ L. H. Li, M. Mexis, P. Ridha, M. Bozkurt, G. Patriarche, P. M. Smowton, P. Blood, P. M. Koenraad, and A. Fiore, Appl. Phys. Lett. 95, 221116 (2009).

${ }^{26}$ Y. H. Huo, B. J. Witek, S. Kumar, J. R. Cardenas, J. X. Zhang, N. Akopian, R. Singh, E. Zallo, R. Grifone, D. Kriegner, R. Trotta, F. Ding, J. Stangl, V. Zwiller, G. Bester, A. Rastelli, and O. G. Schmidt, Nat. Phys. 10, 46 (2014).

${ }^{27}$ A. Muller, W. Fang, J. Lawall, and G. S. Solomon, Phys. Rev. Lett. 103, 217402 (2009).

${ }^{28}$ M. Zieliński, J. Phys.: Condens. Matter 25, 465301 (2013).

${ }^{29}$ L. H. Li, G. Patriarche, and A. Fiore, J. Appl. Phys. 104, 113522 (2008).

${ }^{30}$ L. H. Li, P. Ridha, G. Patriarche, N. Chauvin, and A. Fiore, Appl. Phys. Lett. 92, 121102 (2008).

${ }^{31}$ R. H. Brown and R. Q. Twiss, Nature (London) 177, 27 (1956).

${ }^{32}$ B. J. Witek, R. W. Heeres, U. Perinetti, E. P. A. M. Bakkers, L. P. Kouwenhoven, and V. Zwiller, Phys. Rev. B 84, 195305 (2011).

${ }^{33}$ Ł. Dusanowski, A. Musiał, A. Maryński, P. Mrowiński, J. Andrzejewski, P. Machnikowski, J. Misiewicz, A. Somers, S. Höfling, J. P. Reithmaier, and G. Sęk, Phys. Rev. B 90, 125424 (2014).

${ }^{34}$ R. Brouri, A. Beveratos, J. P. Poizat, and P. Grangier, Opt. Lett. 25, 1294-1296 (2000).

${ }^{35}$ Ł. Dusanowski, A. Golnik, M. Syperek, M. Nawrocki, G. Se,k, J. Misiewicz, T. W. Schlereth, C. Schneider, S. Höfling, M. Kamp, and A. Forchel, Appl. Phys. Lett. 101, 103108 (2012).

${ }^{36}$ A. Schlehahn, L. Krüger, M. Gschrey, J.-H. Schulze, S. Rodt, A. Strittmatter, T. Heindel, and S. Reitzenstein, Rev. Sci. Instrum. 86, 013113 (2015).

${ }^{37}$ M. Benyoucef, T. Alzoubi, J. P. Reithmaier, M. Wu, and A. Trampert, Phys. Status Solidi A 211, 817 (2013).

${ }^{38}$ D. Bordel, M. Rajesh, M. Nishioka, E. Augendre, L. Clavelier, D. Guimard, and Y. Arakawa, Physica E 42, 2765 (2010).

${ }^{39}$ C. W. Pei, J. B. Héroux, J. Sweet, W. I. Wang, J. Chen, and M. F. Chang, J. Vac. Sci. Technol., B: Microelectron. Nanometer Struct. 20, 1196 (2002).

${ }^{40}$ H. Subbaraman, X. Xu, A. Hosseini, X. Zhang, Y. Zhang, D. Kwong, and R. T. Chen, Opt. Express 23, 2487 (2015).

${ }^{41}$ X. Zhang, M. Tomes, and T. Carmon, Opt. Express 19, 9066 (2011).

${ }^{42}$ P. M. Intallura, M. B. Ward, O. Z. Karimov, Z. L. Yuan, P. See, P. Atkinson, D. A. Ritchie, and A. J. Shields, J. Opt. A: Pure Appl. Opt. 11, 054005 (2009). 